\title{
NERVOUS SYMPTOMS SECONDARY TO SLIGHT GASTRIC DISTURBANCE.
}

BY s. G. WEBBER, M. D.

IT is by no means always easy to unravel the complicated relations of cause and effect even in apparently very trivial cases. The viscera, above all the abdominal viscera, are not abundantly supplied with sensitive nerves, and disturbance of function, unaccompanied by organic lesion, may give rise to so slight local symptoms that the patient entirely overlooks them; especially is this true if other secondary symptoms, producing great discomfort, mask the primary affection. It is chiefly or only in the comparatively trifling affections of the viscera that this is true; serious lesions are revealed by such marked symptoms that they are not likely to be overlooked.

The manner in which the secondary symptoms are produced must be varied. The quality of the blood may be changed and hence the nutrition of distant organs may suffer; there is loss of vigor, or actual structural change. This is the case in kidney disease ; cerebral symptoms may be the first to attract attention; the blood is poisoned by retained excrementitious substances. The same may be true in disturbances of the digestive organs from the retention or reabsorption of excretions.

But there is less liability to error of diagnosis in these cases than there is in another class, wherein there seems to be no blood poisoning and where there is little or no derangement of nutrition. It appears reasonable to believe that often the nervous system furnishes the path by which the abnormal influence is conveyed to distant organs; whether discomfort is caused by the action of the vaso-motor nerves upon the blood-vessels, or by an irritation of the nerve centres themselves and thereby through reflex influence, it may be impossible to decide in every case.

Let the afferent nerve, whether sympathetic, pneumogastric, or spinal, convey an irritation to the central organ, and that irritation, if of sufficient strength, may in turn arouse the nerve centre of a different region, and the secondary irritation, experienced in a nervous centre which is more irritable or less stable than that originally excited, is manifested by spasmodic action or by a sense of discomfort which may amount to severe pain. Yet the primary irritation is not sufficiently strong to awake consciousness. There is nothing new in this, and my object is merely to call attention to two or three characteristic cases, patients who came to me or were sent solely on account of nervous disturbances without any attempt having been made to regulate the digestion. It is not strange if we sometimes overlook disorders which produce no symp- 
toms, or where their symptoms are so thrown into the shade that the patient takes no thought of them. In none of the cases to which I shall refer did the patients themselves give more than a secondary importance to the derangements of the digestive organs.

Headaches and other cerebral symptoms are common after irregularities in diet, but Anstie says that it is " exceedingly rare for irritation conveyed from the alimentary canal to take any important part in setting up neuralgia of a distant nerve." He refers to Valleix as making the same statement in case of neuralgias of the head. Other authors include gastric and intestinal irritation among the causes of neuralgia.

In the case of a patient affected with neuralgia, the pain shifting from one region to another, now in the legs, now in the back, arms, face, or head, with each attack forsaking the former locality and appearing elsewhere, I was led finally to refer the pain to gastric disturbance as a cause. Tonics and various anti-neuralgic remedies proved only temporarily of benefit; at length it was noticed that there was during each attack a sense of abdominal fullness. There was little or no distress after eating and only occasionally undue acidity of the stomach. Various remedies were then used with a view to correcting the disease of the digestive organs. Those directed to the stomach, such as quinine, pepsin, mineral acids, and charcoal, gave a temporary relief only, and it was necessary to change from one to the other, but during this time it was difficult to persuade the patient to carry out the directions given about exercise ${ }^{\prime}$ and diet. Finally small doses of calomel and strychnia gave more favorable results, in part, perhaps, because more care was used by the patient in regard to both exercise and diet. After this there was a very great improvement, the neuralgic attacks were much less frequent and intense, and none of any severity have been experienced for several months.

In this case the coincidence of neuralgia with flatulence and the fact that both yielded when attention was directed to the digestive organs would show that the neuralgia was secondary to the dyspepsia. Yet it is certainly true that there was a condition ready to develop neuralgia in the patient's state of health.

- And so in other cases of nervous disturbance secondary to disease of the viscera there is probably a state favorable to the appearance of the secondary symptoms. In the following case excessive smoking combined with hard work and mental worry to develop the required condition. That hard work and mental worry were only predisposing causes may be inferred from the fact that they continued to act after tobacco was given up and the stomach was cared for, but the nervous symptoms did not return.

The patient was a lawyer who was troubled with distressing sensations, pressure at the occiput, slight pain in the forehead, sometimes 
dizziness, tingling in various parts, mental lassitude, a tired feeling, and " the blues." There was also a sense of oppression at the epigastrium, with acid eructations and belching of wind. At times the heart beat violently, and there was a slight pain in the cardiac region; there was no pain in shoulder or in arm. He smoked one cigar in the morning, one at noon, and several in the evening; he also drank strong tea and coffee. Under treatment directed to the digestive organs and by diminishing the number of cigars to one a day, which he cut into three pieces to smoke at the usual hours, he greatly improved. Several months later there was a little discomfort on lying down, especially if the stomach was full. This finally disappeared.

In another case the gastric disturbance was not so well marked, yet I have no doubt it was one chief cause of the cerebral symptoms. A lawyer overworked himself, neglected exercise, was irregular at his meals, ate rapidly, and went to work immediately after. $\mathrm{He}$ suffered from headache, dizziness, and various unpleasant sensations, weakness and loss of mental power. None of the symptoms were very severe, but combined they nearly deprived the patient of the power of working. He thought his digestion was good, and was not aware of any dyspeptic symptoms, but at times there was a slight nausea, and the urine immediately after passing was cloudy from a granular deposit. Subsequently, perhaps because his attention had been called to the stomach, he noticed more decided dyspeptic symptoms. It was by no means easy to separate the effects of overwork from those of indigestion, but I was strongly inclined to believe that taking hurried and irregular meals at eating-houses, with food probably not too well cooked, was really the cause that the hard work proved injurious. This patient was rather unmanageable: he found it difficult to take twenty minutes for dinner; exercise was a burden to him; he could not endure taking medicine regularly. Nevertheless, with a short vacation, quinia, extract of nux vomica, and hydrochloric acid the urine cleared up, the headache ceased, and his condition greatly improved, but during the last three years he has repeatedly disregarded cautions and has had a return of the old symptoms.

A patient now under treatment illustrates more strikingly than those just referred to the connection between cerebral symptoms and irregularities in the digestive organs. But there was also overwork and anxiety, with disregard of all hygienic rules so far as concerns eating. The dyspeptic symptoms first appeared and were not heeded until cerebral symptoms were developed.

But because there are cerebral and gastric symptoms coexisting one must not jump at the conclusion that the latter are the cause of the former. Just the opposite may be the case. Most cases of sick headache are purely nervous in origin. Many patients refer them to de- 
rangement of the stomach, probably because there is vomiting and because imprudence in diet is an exciting cause of an attack; but other disturbing influences, as severe mental application, loss of sleep, etc., may cause an attack independently of all imprudence in diet. Thus a patient who had been troubled with attacks of vertigo, sometimes with vomiting, often with nausea, found that attacks were caused by eating fried food, sitting up late, attending concerts, a strong wind from a certain point of the compass, noises, a bright sun, and by looking at certain motions as that of the waves. There was a similarity to epilepsy in the attacks, but a stronger resemblance to migraine. There was apparently no indigestion; between the attacks the health was excellent; there was no heart-burn, no acidity of stomach, no flatulence. Remedies directed to improving the digestive powers did little or no good ; strychnia seemed to aggravate the condition; bromide of potassium alone, or combined with the iodide, and blistering the back of the neck were beneficial.

The difference between such cases as the last and the others is great. In the former the dyspeptic symptoms may be insignificant, but they either exist before the nervous symptoms or are present continuously; no other adequate cause can be discovered to account for the disturbance of the health; remedies directed to correct the digestive irregularities are beneficial while other remedies fail. In those cases where the nervous system is at fault primarily, the digestive symptoms are not noticed before the nervous, and where the attacks are periodical they are not present in the intervals; other influences than imprudence in diet will cause an attack; the symptoms are more frequently nausea and vomiting, which by its severity obtrudes itself into notice; remedies directed to the nervous system give the best results. Unfortunately, overwork, anxiety, or care as predisposing causes may give rise to either purely nervous disturbance or to nervous weakness and gastric derangement, leading then to such attacks as have been described, and this renders a diagnosis difficult.

\section{RECENT PROGRESS IN THERAPEUTICS. ${ }^{2}$}

BY ROBERT AMORY, M. D.

Effects of Saline Cathartics, Nitro-Muriatic Acid, and Mercury upon the Bile. ${ }^{2}$ - In continuing these experiments (vide previous reports) Professor Rutherford has taken up the consideration of those substances which are more commonly found in natural spring waters having cathartic properties. His results may be summarized :-

1 Concluded from page 343.

${ }^{2}$ Experiments on the Biliary Secretion of Dogs: Rutherford and Vignal. Journal of Anatomy and Physiology, July, 1877. 